\title{
Twenty-four-hour ambulatory blood pressure and duration of hypertension as major determinants for intima-media thickness and atherosclerosis of carotid arteries
}

\author{
Ta-Chen Su ${ }^{\mathrm{a}, \mathrm{b}}$, Yuan-Teh Lee ${ }^{\mathrm{a}}$, Suzzana Chou ${ }^{\mathrm{b}}$, Wen-Tsan Hwang ${ }^{\mathrm{b}}$, \\ Chen-Fang Chen ${ }^{a, b}$, Jung-Der Wang ${ }^{a, b, *}$ \\ ${ }^{a}$ Department of Internal Medicine, National Taiwan University Hospital, Taipei, Taiwan \\ ${ }^{\mathrm{b}}$ Institute of Occupational Medicine and Industrial Hygiene, College of Public Health, National Taiwan University, \\ Rm 1505, No. 1, Jen-Ai Rd., 1st Sec., Taipei 100, Taiwan
}

Received 21 November 2004; received in revised form 13 March 2005; accepted 28 March 2005

Available online 1 June 2005

\begin{abstract}
The relationship between time factors of elevated blood pressure (BP) and carotid atherosclerosis (CA) is still unclear. The associations between time-weighted average $24 \mathrm{~h}$ ambulatory systolic BP (TWA-SBP), duration of hypertension in years (hypertension-year), and CA were investigated in a petrochemical company sample of 95 executives and 91 gender- and age-matched non-executives employees. Intima-media thickness (IMT) and plaque scores of extracranial carotid artery (ECCA) were determined bilaterally by high-resolution B-mode ultrasound. The determinants of segment-specific carotid IMT and odds ratios for CA, in terms of thicker IMT (IMT $\geq 75$ th percentile) and ECCA score $\geq 3$, were evaluated by multivariate regression analysis. Results revealed TWA-SBP and hypertension-year were two major determinants of IMT at common carotid artery (CCA) and carotid bulb by using mixed regression models. However, TWA-DBP was a negative determinant of IMT at CCA and carotid bulb. Meanwhile, the executives were found to be a negative association with IMT at carotid bulb. Measurements at both internal carotid artery and bulb identified duration of diabetes mellitus as significant determinant of IMT. After controlling covariates, multivariate logistic regression analysis identified TWA-SBP and hypertension-year as the important determinants for thicker IMT and ECCA 23. And, TWA-DBP was found as a negative determinant for CA. In conclusion, both TWA-SBP and hypertension-year were two major determinants for carotid IMT and CA, which seem to imply that both short-term and long-term durations of elevated BP are probably crucial in the pathogenesis of CA.
\end{abstract}

(C) 2005 Elsevier Ireland Ltd. All rights reserved.

Keywords: Ambulatory blood pressure; Hypertension; Time-weighted average; Intima-media thickness; Carotid atherosclerosis

\section{Introduction}

Elevated blood pressure (BP) is one of the most important risk factors in the development of atherosclerosis and its related cardiovascular disease (CVD) [1]. Carotid arteries intima-media thickness (IMT) and extracranial carotid artery (ECCA) atherosclerosis assessed by noninvasive ultrasonography have been demonstrated as good predictors for CVD

\footnotetext{
* Corresponding author. Tel.: +886 223516561; fax: +8862 23224660.

E-mail address: jdwang@ha.mc.ntu.edu.tw (J.-D. Wang).
}

morbidity and mortality $[2,3]$. We also have documented that hypertension plays a major role in the pathogenesis of carotid atherosclerosis (CA) in a community-based population [4], and CA has been strongly associated with stroke in a Chinese hospital-based study [5].

In comparison to casual BP checkup at a doctor's office, the measure of ambulatory $\mathrm{BP}(\mathrm{AmBP})$ is a more accurate reflection of the dynamic $\mathrm{BP}$ change during a typical day and is probably a more suitable predictor for long term damage to target organs and clinical prognosis, including morbidity and mortality related to CVD [6,7]. 
This study was designed to evaluate the associations between time-weighted average $24 \mathrm{~h}$ ambulatory systolic BP (TWA-SBP), duration of hypertension in years (hypertension-year), and CA in executives and nonexecutives employees. The determinants of segment-specific carotid artery IMT and odds ratios for CA were also estimated after control of potential confounders.

\section{Methods}

\subsection{Subjects}

From July to December 2002, we invited 200 employees to participate in this cardiovascular examination for a health promotion program in a petrochemical company in Taipei. The program recruited 100 executives (nearly $90 \%$ of this Taipei office) who were above the rank of deputy director of a department and another 100 age- and gendermatched non-executive employees. We performed a carotid ultrasonography and a $24 \mathrm{~h} \mathrm{AmBP}$ monitoring for each participant.

\subsection{Medical history and definitions}

BP measurements were performed with a mercury sphygmomanometer in a standardized fashion. Systolic and diastolic BP were defined according to Korotkoff sounds I and V. Subjects with SBP higher than $140 \mathrm{mmHg}$ and/or diastolic BP (DBP) higher than $90 \mathrm{mmHg}$, or receiving anti-hypertension agents were considered to be hypertensive. Prevalent diabetes mellitus (DM) was defined as fasting glucose $\geq 126 \mathrm{mg} / \mathrm{dL}$, and/or a history of DM with management. Hypertension-year or DM-year was defined as the total duration from the year of hypertension or DM first diagnosed until this examination date, which were verified by reviewing every subject's medical chart. Body mass index was computed from the subject's weight $(\mathrm{kg})$ over height $(\mathrm{m})$ squared. Data on alcohol use and smoking were obtained for each subject from a self-reported questionnaire. The ethics committee of National Taiwan University Hospital has approved this study and an informed consent was obtained from each study subject before study.

\subsection{The 24-h ambulatory blood pressure measurements}

Twenty-four-hour BP monitoring was recorded from cuff sphygmomanometer of an oscillometric BP device using Dynapulse 5000 (Pulse Metric Inc., San Diago, CA, USA). From this device, $\mathrm{BP}$ was determined by examing pressure waveform changes due to Bernoulli flow effects. This BP measurement method was validated against invasive measurement [8] and noninvasive measurements [9]. TWA-SBP and TWA-DBP were used as the BP components in the analysis. Daytime monitoring was set during 7 a.m. to 10 p.m. with consecutive measurements every $15 \mathrm{~min}$, and nighttime $\mathrm{BP}$ was measured during 10 p.m. to 7 a.m. every 30 min. Thus, TWA-BP was defined as the following formula:

24-h BP $=\frac{\left.2 \sum_{i=1}^{k} \mathrm{BP}_{i}(\text { nighttime })+\sum_{j=1}^{l} \mathrm{BP}_{j} \text { (daytime }\right)}{2 k+l}$

Number of measurements at daytime was $l$ and nighttime was $k$.

\subsection{ECCA ultrasonographic measurement}

A Hewlett-Packard SONO 4500 ultrasound system (Andover, MA, USA), equipped with a 3-11 MHz real-time Bmode scanner, was used for the evaluation. Carotid end-organ disease was assessed by maximal IMT at carotid arteries and by ECCA plaque score. Two measures of maximal IMT at the CCA $0-20 \mathrm{~mm}$ proximal to the carotid bifurcation were obtained bilaterally. One measure of maximal IMT each at carotid bulb and internal carotid artery (ICA) were also obtained bilaterally. For future and subsequent off-line analysis, all scans were recorded on super-VHS videotape. The intraclass correlation coefficients of intra-observer were about $0.70-0.87$ for both sides of CCA IMT measurements as reported previously [10].

The plaque scoring quantified method has been described previously $[4,5,10]$. In brief, a focal thickening of IMT with $>50 \%$ of thickness than adjacent IMT was considered as an atherosclerotic plaque. A grade was assigned for each chosen segment: grade 0 for normal or no observable plaque, grade 1 for one small plaque with diameter stenosis $<30 \%$, grade 2 for one medium plaque with 30-49\% diameter stenosis or multiple small plaques, grade 3 for one large plaque with $50-99 \%$ diameter stenosis or multiple plaques with at least one medium plaque, and grade 4 for $100 \%$ occlusion. Carotid artery segments, including the proximal and distal CCA (>20 mm and $0-20 \mathrm{~mm}$ distal to the bulb bifurcation, respectively), bulb, internal carotid artery, and external carotid artery, were examined bilaterally. The plaque score was calculated by summing the plaque grades at 10 segments of the ECCA. Reproducibility of the plaque grade scoring expresses good agreement with a kappa value of 0.70 $[4,5]$.

\subsection{Lipids, glucose and $C$-reactive protein assays}

Fasted blood samples (10-14h overnight) were drawn from antecubital vein for determinations of lipids and glucose with subjects in a seated position. Serum levels of lipids, including total cholesterol (TC), high-density lipoprotein cholesterol (HDL-C), and triglyceride were assayed in the central laboratory of the National Taiwan University Hospital. Low-density lipoprotein cholesterol (LDL-C) was calculated from Friedewald's formula. Levels of blood glucose were determined by routine enzymatic method. Serum high sensitive C-reactive protein (hs-CRP) was measured by us- 
ing a chemiluminescent enzyme-labeled immunometric assay (Diagnostic Products Co., Los Angeles).

\subsection{Statistical analyses}

In the data analysis, clinical features and cardiovascular risk factors of study subjects was first compared between executives and non-executives. Continuous variables were expressed as the mean \pm 1 standard deviation. The $t$-test was used to make comparisons for continuous variables between the two groups. For categorical data, Chi-square test was used to test the significance level between the two groups. The average IMT measurements at CCA, bulb and ICA on both sides, as well as ECCA plaque scores, were compared by executive status.

The determinants of IMT at different locations of carotid arteries were analyzed by constructing mixed regression models. The pressure load obtained from AmBP measurements, expressed as TWA-SBP and TWA-DBP, was also put into the mixed regression models. The strength of associations between CA and potential risk factors was explored in terms of odds ratios (OR) in the $95 \%$ confidence interval $(95 \% \mathrm{CI}$ ) using multivariate logistic regression (MLR) analysis. In addition to the segment-specific IMT correlation analysis, the average IMT, which was the representative of the general aspects of carotid structural changes, also was put in MLR models. The upper quartile $(\geq 75$ th percentile) of IMT value, which was obtained from the mean value of 8 IMT measurements at different locations of carotid arteries for each participant, was considered as a thicker IMT. And ECCA plaque score equal to or above 3 (around upper quartile) was considered as a significant CA.

\section{Results}

Among participants, two cases refused AmBP study, and incomplete measurement in 12 other subjects. We used the results of AmBP if the valid measurements were more than three-fourths of total measurements periods during daytime and nighttime. Thus, we have successfully obtained 186 cases with complete data of AmBP and carotid duplex measurements. Table 1 summarized the baseline characteristics of participants; there was no significant difference between these two groups.

In Table 2, the results of measurements at carotid IMTs and ECCA plaque scores in different locations of carotid arteries revealed no significant difference between executives and non-executives. Mixed regression models on Table 3 demonstrated segment-specific determinants of carotid IMT. Because the duration of hypertension fit much better than simple classification of a positive history of hypertension in IMT at all locations of carotid arteries (CCA, bulb and ICA), we presented only the models with hypertension-year. Model 1 used TWA-SBP and TWA-DBP to represent the effect of BP
Table 1

Baseline characteristics of executives and non-executives employees in a petrochemical company

\begin{tabular}{llll}
\hline Characteristics & $\begin{array}{l}\text { Executives } \\
(n=95)\end{array}$ & $\begin{array}{l}\text { Non-executives } \\
(n=91)\end{array}$ & $P$-value \\
\hline Age (years) & $55.6 \pm 5.3$ & $55.8 \pm 5.1$ & 0.81 \\
Male (\%) & 87.4 & 87.9 & 0.91 \\
Hypertension (\%) & 28.4 & 22.0 & 0.39 \\
TWA SBP (mmHg) & $124.6 \pm 10.6$ & $123.0 \pm 10.8$ & 0.34 \\
TWA DBP (mmHg) & $72.5 \pm 7.2$ & $72.0 \pm 6.7$ & 0.60 \\
Diabetes mellitus (\%) & 9.5 & 6.6 & 0.31 \\
Smoker, current (\%) & 23.1 & 16.8 & 0.29 \\
Alcohol habit $(\%)^{\mathrm{a}}$ & 13.2 & 22.1 & 0.11 \\
LDL-C (mmol/L) & $3.3 \pm 0.9$ & $3.4 \pm 0.9$ & 0.44 \\
HDL-C (mmol/L) & $1.4 \pm 0.3$ & $1.4 \pm 0.3$ & 0.60 \\
BMI (kg/m $\left.{ }^{2}\right)$ & $24.3 \pm 2.8$ & $24.0 \pm 2.6$ & 0.41 \\
\hline
\end{tabular}

Low-density lipoprotein cholesterol, LDL-C; high-density lipoprotein cholesterol, HDL-C; body mass index, BMI.

${ }^{a}$ Alcohol habit: regular alcohol drinking at least one drink per week.

components, while model 2 used casual office BP for comparison. Both models showed that LDL-C were significant determinant of IMT at CCA. TWA-SBP, hypertension-year and age were the major determinant of IMT at CCA and bulb, but not at ICA. And, TWA-DBP was found as a negative determinant for carotid IMT at CCA and bulb. However, casual office BP was not demonstrated as a significant determinant of IMTs at all ECCA. Being an executive also was a negative determinant for IMT at bulb. DM-year was found to be a significant determinant for IMT at ICA and bulb. However, inflammation marker of hs-CRP was found not to be a significant predictor for all carotid IMTs in this study.

After covariates adjustment, MLR analysis revealed that hypertension-year, TWA-SBP, and age increased the risk of both for a thicker IMT and an ECCA score $\geq 3$ as shown in Table 4. And, TWA-DBP was found as a negative determinant

Table 2

Carotid intima-media thickness and extracranial carotid artery (ECCA) plaque score in different locations of carotid arteries

\begin{tabular}{cllll}
\hline & & Executive & Non-executives & $P$-value \\
\hline IMT $(\mathrm{mm})$ & & & & \\
CCA & Right & $0.76 \pm 0.17$ & $0.79 \pm 0.25$ & 0.30 \\
& Left & $0.81 \pm 0.21$ & $0.83 \pm 0.28$ & 0.69 \\
Bulb & Right & $0.95 \pm 0.42$ & $1.04 \pm 0.51$ & 0.17 \\
& Left & $0.96 \pm 0.43$ & $1.07 \pm 0.49$ & 0.09 \\
ICA & Right & $0.72 \pm 0.34$ & $0.68 \pm 0.35$ & 0.45 \\
& Left & $0.65 \pm 0.18$ & $0.67 \pm 0.22$ & 0.45 \\
ECCA Plaque & score & & & \\
CCA & Right & $0.12 \pm 0.31$ & $0.05 \pm 0.30$ & 0.82 \\
& Left & $0.09 \pm 0.33$ & $0.11 \pm 0.44$ & 0.47 \\
Bulb & Right & $0.45 \pm 0.76$ & $0.62 \pm 0.86$ & 0.14 \\
& Left & $0.42 \pm 0.70$ & $0.59 \pm 0.88$ & 0.13 \\
ICA & Right & $0.13 \pm 0.42$ & $0.11 \pm 0.43$ & 0.74 \\
& Left & $0.04 \pm 0.24$ & $0.08 \pm 0.31$ & 0.31 \\
\hline
\end{tabular}

CCA, common carotid artery; ICA, internal carotid artery. 
Table 3

Predictive effects of various determinants of intima-media thickness at different locations of carotid arteries

\begin{tabular}{|c|c|c|c|c|c|c|}
\hline \multirow[t]{2}{*}{ Variables } & \multicolumn{2}{|c|}{ Common carotid artery } & \multicolumn{2}{|l|}{ Carotid bulb } & \multicolumn{2}{|c|}{ Internal carotid artery } \\
\hline & $\begin{array}{l}\text { Model 1: } \beta \pm \\
\text { S.E. } \times 10^{-3}(\mathrm{~mm})\end{array}$ & $\begin{array}{l}\text { Model 2: } \beta \pm \\
\text { S.E. } \times 10^{-3}(\mathrm{~mm})\end{array}$ & $\begin{array}{l}\text { Model 1: } \beta \pm \\
\text { S.E. } \times 10^{-3}(\mathrm{~mm})\end{array}$ & $\begin{array}{l}\text { Model 2: } \beta \pm \\
\text { S.E. } \times 10^{-3}(\mathrm{~mm})\end{array}$ & $\begin{array}{l}\text { Model 1: } \beta \pm \\
\text { S.E. } \times 10^{-3}(\mathrm{~mm})\end{array}$ & $\begin{array}{l}\text { Model 2: } \beta \pm \\
\text { S.E. } \times 10^{-3}(\mathrm{~mm})\end{array}$ \\
\hline Intercept & $-26.18 \pm 27.50$ & $-1.66 \pm 24.69$ & $-35.78 \pm 46.73$ & $-18.68 \pm 42.20$ & $27.75 \pm 30.00$ & $34.77 \pm 26.26$ \\
\hline $\mathrm{SBP}(\mathrm{mmHg})$ & $0.84 \pm 0.27^{\ddagger}$ & $0.18 \pm 0.13$ & $1.47 \pm 0.47^{\ddagger}$ & $-0.06 \pm 0.22$ & $0.39 \pm 0.30$ & $0.19 \pm 0.14$ \\
\hline $\mathrm{DBP}(\mathrm{mmHg})$ & $-0.94 \pm 0.43^{*}$ & $-0.20 \pm 0.23$ & $-2.03 \pm 0.74^{\dagger}$ & $0.12 \pm 0.39$ & $-0.64 \pm 0.47$ & $-0.38 \pm 0.24$ \\
\hline Hypertension-year & $0.90 \pm 0.36^{*}$ & $0.86 \pm 0.37^{*}$ & $2.62 \pm 0.61^{\ddagger}$ & $2.52 \pm 0.63^{\ddagger}$ & $0.72 \pm 0.39$ & $0.70 \pm 0.39$ \\
\hline Hypertension-tx & $3.59 \pm 4.42$ & $4.34 \pm 4.51$ & $-13.11 \pm 7.51$ & $-11.04 \pm 7.71$ & $-2.76 \pm 4.82$ & $-3.25 \pm 4.80$ \\
\hline DM-year & $0.02 \pm 0.60$ & $-0.03 \pm 0.64$ & $2.16 \pm 1.02^{*}$ & $2.51 \pm 1.09^{*}$ & $1.47 \pm 0.66^{*}$ & $1.24 \pm 0.68$ \\
\hline Smoke, pack-year & $0.15 \pm 0.14$ & $0.15 \pm 0.14$ & $0.25 \pm 0.24$ & $0.28 \pm 0.25$ & $0.07 \pm 0.15$ & $0.06 \pm 0.15$ \\
\hline Age (years) & $0.98 \pm 0.30^{\ddagger}$ & $1.06 \pm 0.31^{\ddagger}$ & $1.31 \pm 0.51^{*}$ & $1.62 \pm 0.52^{\ddagger}$ & $0.50 \pm 0.33$ & $0.50 \pm 0.33$ \\
\hline hs-CRP (mg/mL) & $-5.10 \pm 6.70$ & $-5.14 \pm 6.88$ & $-6.68 \pm 11.38$ & $-5.58 \pm 11.75$ & $-2.04 \pm 7.31$ & $-2.76 \pm 7.32$ \\
\hline LDL-C (mmol/L) & $3.42 \pm 1.66^{*}$ & $3.51 \pm 1.70^{*}$ & $-1.12 \pm 2.81$ & $-0.97 \pm 2.91$ & $-0.89 \pm 1.81$ & $-0.89 \pm 1.81$ \\
\hline $\mathrm{HDL}-\mathrm{C}(\mathrm{mmol} / \mathrm{L})$ & $2.66 \pm 5.06$ & $3.91 \pm 5.26$ & $3.43 \pm 8.60$ & $3.26 \pm 8.99$ & $8.01 \pm 5.52$ & $9.29 \pm 5.59$ \\
\hline Male & $-1.12 \pm 5.13$ & $-0.49 \pm 5.38$ & $10.85 \pm 8.73$ & $10.40 \pm 9.20$ & $4.65 \pm 5.60$ & $5.56 \pm 5.72$ \\
\hline $\mathrm{BMI} \geqslant 25\left(\mathrm{Kg} / \mathrm{m}^{2}\right)$ & $2.54 \pm 3.21$ & $2.33 \pm 3.29$ & $-7.14 \pm 5.46$ & $-8.74 \pm 5.62$ & $3.83 \pm 3.51$ & $3.76 \pm 3.49$ \\
\hline Executives & $-3.92 \pm 3.01$ & $-3.30 \pm 3.08$ & $-11.55 \pm 5.11^{*}$ & $-11.16 \pm 5.27^{*}$ & $-0.78 \pm 3.28$ & $-0.33 \pm 3.28$ \\
\hline AIC & -3019.2 & -3009.2 & -1087.2 & -1075.2 & -1365.3 & -1363.6 \\
\hline
\end{tabular}

Analysis by using mixed models; model 1, means adjusted cofactors of time-weighted average SBP and DBP; model 2, means adjusted cofactors of office SBP and DBP. Hypertension-year, duration of hypertension in years; Hypertension-tx, hypertension under treatment; hs-CRP: high sensitive C-reactive protein; HDL-C: high-density lipoprotein cholesterol; LDL-C: low-density lipoprotein cholesterol; BMI: body mass index; AIC: Akaike's Information Criterion.

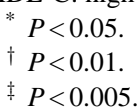

for thicker IMT and ECCA score $\geq 3$. The inclusion of TWASBP and TWA-DBP fit better than simple office SBP and DBP after putting hypertension-year in the models for thicker IMT and ECCA score $\geq 3$.

\section{Table 4}

Multivariate logistic regression analysis for risk factors of thicker carotid intima-media thickness (IMT) and carotid atherosclerosis

\begin{tabular}{lll}
\hline Characteristics & $\begin{array}{l}\text { IMT } \geq 75 \text { th } \\
\text { percentile: OR }(95 \% \\
\text { CI) }\end{array}$ & $\begin{array}{l}\text { ECCA score } \geq 3: \\
\text { OR }(95 \% \text { CI })\end{array}$ \\
\hline Model 1 & & \\
Executives & $0.48(0.21-1.10)$ & $0.70(0.30-1.65)$ \\
Hypertension-year & $1.12(1.03-1.22)^{*}$ & $1.16(1.05-1.27)^{\ddagger}$ \\
Hypertension-tx & $0.84(0.26-2.73)$ & $0.70(0.20-2.46)$ \\
TWA-SBP (mmHg) & $1.16(1.07-1.27)^{\ddagger}$ & $1.16(1.06-1.27)^{\ddagger}$ \\
TWA-DBP (mmHg) & $0.82(0.72-0.93)^{\ddagger}$ & $0.87(0.76-0.99)^{*}$ \\
Age (years) & $1.11(1.02-1.22)^{*}$ & $1.16(1.05-1.28)^{\ddagger}$ \\
DM-year & $1.16(0.95-1.41)$ & $1.24(0.99-1.54)$ \\
Model 2 & & \\
Executives & $0.55(0.25-1.21)$ & $0.76(0.33-1.76)$ \\
Hypertension-year & $1.09(1.01-1.19)^{*}$ & $1.13(1.03-1.24)^{\dagger}$ \\
Hypertension-tx & $0.96(0.32-2.86)$ & $0.81(0.25-2.64)$ \\
Office SBP (mmHg) & $1.02(0.99-1.05)$ & $1.03(1.00-1.07)$ \\
Office DBP (mmHg) & $1.00(0.94-1.05)$ & $0.98(0.93-1.04)$ \\
Age (years) & $1.13(1.03-1.23)^{\dagger}$ & $1.16(1.05-1.28)^{\ddagger}$ \\
DM-year & $1.15(0.96-1.38)$ & $1.22(0.98-1.48)$ \\
\hline All
\end{tabular}

All models were adjusted for above variables and male gender, HDL-C, LDL-C, pack-year of smoking and BMI $\geq 25$. Hypertension-year, duration of hypertension in years; hypertension-tx, hypertension under treatment; TWA, time-weighted average; SBP, systolic blood pressure; DBP, diastolic blood pressure.

$$
\begin{aligned}
& * P<0.05 . \\
& \dagger P<0.01 . \\
& { }^{\dagger} P<0.005 .
\end{aligned}
$$

\section{Discussions}

This study demonstrated TWA-SBP and hypertensionyear to be two major determinants of carotid IMT and CA in middle-aged white-collar workers after controlling potential confounders. Both measurements imply that the significance of time factor should be taken into consideration for estimating the increased workload of cardiovascular system by higher BP. All these findings require careful scrutiny before reaching any conclusions.

\subsection{Duration of hypertension and $C A$}

Subjects with hypertension might not necessarily be under adequate control. A detailed inquiry into their medication history found that only about $67 \%$ of 47 subjects with hypertension regularly received antihypertensive treatment and addition of a treatment term for hypertension in the multivariate regression model could not contribute any more significant effect on IMT and CA. In fact, duration of hypertension was reported to be the major risk factor for cerebral white matter lesions [11]. Likewise; we also found that quantitative measures of the duration of diabetes or DM-year were statistically significant determinant for IMT at both bulb and ICA. Thus, we recommended that duration of a chronic disease should be taken into appropriate consideration if the outcome measurement represents a long-term effect or inadequate control of the disease process is common.

\subsection{The significance of 24-h TWA-BP and $C A$}

This study supported previous report that Am-SBP (or TWA-SBP) played a major role in influencing carotid IMT 
and CA [12]. And, it also added some more evidence to the cumulative effect by using AmBP and time factor in terms of pressure-time index to evaluate left ventricular hypertrophy after a long period of hypertension [13]. In this study, we also identify TWA-DBP as a negative determinant for carotid IMT and CA. The findings seemed to indicate that a combination of increased SBP and decreased DBP, in terms of a wide PP, might increase carotid IMT and CA, as was also reported by other investigators $[14,15]$. A wide PP may increase the hemodynamic barotrauma on the vascular wall and subsequently predisposes detrimental effects on the endothelium [16].

In addition, the implication of TWA-SBP as a major determinant of IMT at CCA and bulb also indicated that casual office BP checkup might not be sensitive enough to detect a latent elevation of BP during sometime in a day. Theoretically, 24-h BP reflects more accurately the workload of the cardiovascular system than casual office BP. In fact, a randomized controlled trial for antihypertensive treatment actually showed that with less intensive drug one could achieve a good BP management through 24-h monitoring than office BP [17]. Because the average BP coming from 24-h ambulatory monitoring simply takes the sum of each BP measurements and divide by the number of recordings, it generally under-weights the BP measurements in the evening or during the sleeping time. Thus, the TWA-SBP represents more accurately than the traditionally defined 24-h average SBP, although there is no major difference in the model fitting in this study for the two slightly different indicators (data not shown).

\subsection{Mixed regression models, multivariate logistic regression analysis and carotid IMT measurements}

To our knowledge, this study also is one of the rare studies [18] that applied the analysis of mixed regression model to relate the multiple carotid IMT measures as repeated measurements with its risk factors, while previous studies usually took the average value of repeated IMT measurements [1-4]. The rational use of mixed model assumed repeated measurements to have a random effect, which corresponds more to the bilateral measurements of IMT at a fixed specific segment for each subjects. Statistically, it was generally more efficient than simply taking the mean and can possibly improve the detection power of limited study subjects. However, to corroborate our findings from linear models, we also assess the average values of different IMT measurements in Table 4 by using logistic models, which derived the similar results with mixed models that TWA-SBP measurements and hypertension-year are the two major determinants of carotid IMT and CA. And, TWA-BP is more closely associated with carotid IMT or CA than casual BP measured at office visits.

\subsection{Segment-specific risk factors in $C A$}

Different risk factors found at different segments of carotid arteries in this study might indicate multiple mechanisms of pathogenesis for site-specific risk factors, which were also found in a recent report [19]. The detrimental effects of LDL$\mathrm{C}$ at CCA, and the job title with executives at carotid bulb found in this study might provide some clues for the pathophysiology of CA in future studies. Carotid arterial bifurcations are characterized by low and oscillatory shear stress due to vascular network architectural constraints and are prone to atherosclerosis [20]. Conversely, the causes of the lower sensitivity of risk factors on IMT at ICA segment may be ascribed to a more stable hemodynamic flow, which is characterized with the low peak systolic pressure and the difficulty in obtaining measurements from ICA in some subjects [21]. However, we still identified DM-year as significant determinant of IMT at ICA and bulb in this study. This study supported short-term and long-term independent effects of hemodynamic and metabolic factors on the pathogenesis of CA [22]. The fact of job title of not being an executive to be a significant factor for increased IMT at the bulb indicated that the job title as a CVD risk factor still await further study.

\subsection{Study limitations}

There are some limitations in this study. A cross-sectional design may limit the inference to a causal relationship. And, making multiple comparisons in the mixed regression analysis may overestimate the association between measured sites and risk factors. However, the MLR analysis provided additional evidence to the short-term and long-term cardiovascular effects of a higher BP on CA, which indicated that such associations might not be likely due to chance.

There is a body of evidence that statin could decrease the progression of atherosclerosis [23]. Thus, we reviewed the medication and medical history for each participant. Hypercholesterolemia was defined as total serum cholesterol $\geq 6.21 \mathrm{mmol} / \mathrm{L}$, of which $22 \%$ of executives and $25 \%$ of non-executive controls fulfilled the criterion. However, only about $20 \%$ of subjects with hypercholesterolemia in each group ever used statin, and most of them did not use it continuously because our National Health Insurance required proof of persistent elevation of serum cholesterol level every three-month to allow for a continuous prescription. Thus, the effects of ever taking statin on carotid atherosclerosis were not apparent as one might expect in this study.

\subsection{Conclusions}

In conclusion, both the TWA-BP monitoring and duration of hypertension were useful to assess the short-term and longterm cardiovascular effects of higher BP. AmBP monitoring has a more advantageous role than office BP measurement in the evaluation of short-term and long-term control of hypertension. Application of noninvasive cardiovascular assessments by using AmBP monitoring and carotid ultrasounds in worksite is feasible. 


\section{Acknowledgments}

We thank Bao-Show Hwang and Chi-Ting Tseng for their assistance in this study. This study was supported by a grant from the National Health Research Institute (NHRI-WX939204PP) and another grant sponsored by the Taiwan Chinese Petroleum Company (2002).

\section{References}

[1] Psaty BM, Furberg CD, Kuller LH, et al. Isolated systolic hypertension and subclinical cardiovascular disease in the elderly: initial findings from the Cardiovascular Health Study. J Am Med Assoc 1992;268:1287-91.

[2] O'Leary DH, Polak JF, Kronmal RA, et al. Carotid-artery intima and media thickness as a risk factor for myocardial infarction and stroke in older adults. N Engl J Med 1999;340:14-22.

[3] Chambless LE, Heiss G, Folsom AR, et al. Association of coronary heart disease incidence with carotid arterial wall thickness and major risk factors: the Atherosclerosis Risk in Communities (ARIC) Study. Am J Epidemiol 1997;146:483-94.

[4] Su TC, Jeng JS, Chien KL, et al. Hypertension status is the major determinant of carotid atherosclerosis: a community-based study in Taiwan. Stroke 2001;32:2265-71.

[5] Jeng JS, Chung MY, Yip PK, Hwang BS, Chang YC. Extracranial carotid atherosclerosis and vascular risk factors in different types of ischemic stroke in Taiwan. Stroke 1994;25:1989-93.

[6] Perloff D, Sokolow M, Cowan R. The prognostic value of ambulatory blood pressure. J Am Med Assoc 1983;249:2792-8.

[7] Verdecchia P, Porcellati C, Schillaci G, et al. Ambulatory blood pressure: An independent predictor of prognosis in essential hypertension. Hypertension 1994;24:793-801.

[8] Brinton TJ, Cotter B, Kailasam MT, et al. Development and validation of a noninvasive method to determine arterial pressure and vascular compliance. Am J Cardiol 1997;80:323-30.

[9] Brinton TJ, Daniel Walls E, Chio SS. Validation of pulse dynamic blood pressure measurement by auscultation. Blood Press Monit 1998;3:121-4

[10] Su TC, Jeng JS, Chien KL, et al. Measurement reliability of common carotid artery intima-media thickness by ultrasonographic assessment. J Med Ultrasound 1999;7:73-9.
[11] de Leeuw FE, de Groot JC, Oudkerk M, et al. Hypertension and cerebral white matter lesions in a prospective cohort study. Brain 2002;125:765-72.

[12] Muiesan ML, Pasini G, Salvetti M, et al. Cardiac and vascular structural changes. Prevalence and relation to ambulatory blood pressure in a middle-aged general population in northern Italy: the Vobarno Study. Hypertension 1996;27:1046-52.

[13] Zakopoulos NA, Toumanidis ST, Barlas GJ, et al. A 'pressure-time index' for assessing the severity of essential hypertension. J Hypertens 1999;17:1387-93.

[14] Lassila HC, Sutton-Tyrrell K, Matthews KA, Wolfson SK, Kuller LH. Prevalence and determinants of carotid atherosclerosis in healthy postmenopausal women. Stroke 1997;28:513-7.

[15] Ryan SM, Waack BJ, Weno BL, Heistad DD. Increases in pulse pressure impair acetylcholine-induced vascular relaxation. Am J Physiol 1995;268:H359-63.

[16] Zureik M, Touboul PJ, Bonithon-Kopp C, et al. Cross-sectional and 4-year longitudinal associations between brachial pulse pressure and common carotid intima-media thickness in a general population. Stroke 1999;30:550-5.

[17] Staessen JA, Byttebier G, Buntinx F, et al. Antihypertensive treatment based on conventional or ambulatory blood pressure measurement: a randomized controlled trial. J Am Med Assoc 1997;278:1065-72.

[18] Lange LA, Bowden DW, Langefeld CD, et al. Heritability of carotid artery intima-media thickness in type 2 diabetes. Stroke 2002;33:1876-81.

[19] Mackinnon AD, Jerrard-Dunne P, Sitzer M, et al. Rates and determinants of site-specific progression of carotid artery intimamedia thickness: the carotid atherosclerosis progression study. Stroke 2004;35:2150-4.

[20] Malek AM, Alper SL, Izumo S. Hemodynamic shear stress and its role in atherosclerosis. J Am Med Assoc 1999;282:203542.

[21] O'Leary DH, Polak JF, Kronmal RA, et al. Thickening of the carotid wall: a marker for atherosclerosis in the elderly? Cardiovascular Health Study Collaborative Research Group. Stroke 1996;27: 224-31.

[22] Reaven GM, Lithell H, Landsberg L. Hypertension and associated metabolic abnormalities - the role of insulin resistance and the sympathoadrenal system. N Engl J Med 1996;334:374-81.

[23] Kang S, Wu Y, Li X. Effects of statin therapy on the progression of carotid atherosclerosis: a systematic review and meta-analysis. Atherosclerosis 2004;177:433-42. 